\title{
Ultrafast modulation of semiconductor lasers through a terahertz field
}

\author{
C.Z. Ning ${ }^{1}$ \\ Computational Quantum Optoelectronics
}

NASA Ames Research Center, Mail Stop T27A-1, Moffett Field, CA 94035

S. Hughes and D.S. Citrin

Semiconductor Optics Theory Group, Department of Physics and Materials

Research Center

P.O. Box 642814

Washington State Lniversity, Pullman, WA 99161-281.1

\begin{abstract}
We demonstrate, by means of numerical simulation, a new mechanism to modulate and switch semiconductor lasers at THz and sub-THz frequency rates. A sinusoidal terahertz field applied to a semiconductor laser heats the electron-hole plasma and consequently modifies the optical susceptibility. This allows an almost linear modulation of the output power of the semiconductor laser and leads to a faithful reproduction of the terahertzficld waveform in the emitted laser intensity.
\end{abstract}

\footnotetext{
'cningộnas.nasa.gov
} 
Semiconductor laser modulation and switching via the pumping current at $\mathrm{GHz}$ rates is based on the so-called relaxation oscillation $[1,2]$. Physically, relaxation oscillation describes the periodic energy exchange between the laser field and semiconductor, which is an interband process dominated by stimulated electron-hole pair recombinations at high pumping level and occurs on a nanosecond time scale. For many applications, modulation and switching at higher rates is highly desirable. While improvements in modulation speed due to quantum well (QW) structure designs and other means have been quite impressive, further improvements beyond terts of $\mathrm{GHz}$ seem to be more difficult. The fundamental limit is placed by the slow interband carrier dynamics. While current modulation has the unique property that allows direct and simple modulation, other means to modulate and switch a diode laser at higher rates are under intensive investigation. Notable examples are switching due to non-equilibrium carrier dynamies under femtosecond optical excitation $[3,4,5]$.

In this letter, we report an alternative scheme for modulating and switching semiconductor lasers by employing THz-frequency electric fields. Recent years have seen a surge of interest on the interaction of TIIz waves with QW's, and THz field generation by semiconductor heterostructures. Moreover, our research is motivated by an experimental demonstration of heating of an clectron gas by applying a THz driving ficld (henceforth denoted by TDF) [6]. The experiment demonstrated that even under strong THz irradiation, the electron gas maintains essentially a heated, thermalized form. Subsequent theoretical works $[7,8,9]$ showed that the heated plasma has an almost sinusoidal temperature variation with time with a frequency given by twice that of the TDF. Additionally the associated gain and refractive index change due to the plasma heating eflect indued by a half-eycle THz field can 
also lead to efficient transient optical modulation and may form the basis for an ultrahigh-speed all-optical switch [9].

The purpose of this work is to demonstrate that a continuous TDF can induce a modulated power output from a semiconductor laser with a temporal modulation that faithfully follows the driving field. We consider a standard edge-emitting semiconductor laser with a $\mathrm{QW}$ active medium. In addition to a standard QW edge-emitting laser geometry, a TDF is applied such that the polarization is in the plane of QW layer. Such a TDF can be applied from a free-space $\mathrm{THz}$ source or by integrating the laser with a photoconductor anrl THz transmission structure. Our theoretical approach starts with the following equation for the carrier distribution function $n_{\mathbf{k}}^{\alpha}$

$$
\left(\frac{\partial}{\partial t}+e \mathbf{F}_{\mathbf{T H z}}(t, \mathbf{r}) \cdot \nabla_{\mathbf{k}}\right) n_{\mathbf{k}}^{\alpha}=\left.\frac{\partial n_{\mathbf{k}}^{c \alpha}}{\partial t}\right|_{c-c}+\left.\frac{\partial n_{\mathbf{k}}^{\alpha}}{\partial t}\right|_{c-p h}+\left.\frac{\partial n_{\mathbf{k}}^{\alpha}}{\partial t}\right|_{s t}+\left.\frac{\partial n_{\mathbf{k}}^{\alpha}}{\partial t}\right|_{r \epsilon s t},
$$

where $\alpha=e(+\mathbf{k}), h(-\mathbf{k})$ for electrons and holes, respectively, and $F_{\mathrm{TH} z}$ is the THz field. The first two terms in the right hand side of Eq. (1) represcnt carrier-carrier (c-c) and carrier-phonon (c-ph) scattering. while the third torm accounts for stimulated recombination and the last term for pumping and other decay processes. We note that the TDF creates highly anisotropic carrier distributions $\left(n_{k_{x}} \neq n_{k_{y}}\right)$ which must be solved for on a two dimensional mesh of $k$ points. Thongh a self-consistent many-body calculation of such anisotropic scattering terms is feasible, it involves a significant computational effort, and thiss we restrict ourselves to the standard relaxation-rate approximations for both of the c-c and c-ph scattering in this paper. The rclaxation times (inverse of the relaxation rates) are $100 \mathrm{fs}$ for $\mathrm{c}-\mathrm{c}$ and $600 \mathrm{fs}$ for (-ph scattering. Many-body interactions are not considered in this paper and the related effects will be discussed in a more detailed investigation. For 
this paper, the $\mathrm{THz}$ waveform is assumed to be sinusoidal. The results, however, carry over to more general temporal waveforms that may be of interest for switching and modulation with a signal field.

For the description of the semiconductor laser, we employ a well-tested rate-equation model except we add additional terms for the TDF-induced plasma-heating effects. Under the rate-equation approximation, which essentially amounts to ignoring the polarization dvnamics of the medium, the basic equations take the form:

$$
\begin{aligned}
\frac{\partial E}{\partial t} & =\frac{i K c}{2 n_{g}} \Gamma \chi(N, T) E-\kappa E \\
\frac{\partial N}{\partial !} & =\frac{J}{{ }^{\prime \prime}(t)}-\gamma_{N} N+\frac{r_{0} c_{b}}{2 \hbar} \chi^{\prime \prime}(N, T) \mid E^{2} .
\end{aligned}
$$

where the symbols have their usual meanings: $J, w$, and $\Gamma$ are the pumping current, active medium thickness, and the confinement factor; $K, n_{g}$, and $\epsilon_{b}$ are the optical wavevector, group index, and background dielectric constant; $\kappa$ is the cavity relaxation constant; $E$ is the slowly varying amplitude of the laser field, and $N=2 \sum_{k} n_{k}^{\alpha} / V$ is the carrier density for electrons and holes which are assumed the same. The optical susceptibility, $x=x^{\prime}+i \chi^{\prime \prime}$, can bo written as (linear approximation) [10]:

$$
\gamma(N, T)=\gamma\left(N_{0}, T_{0}\right)+\frac{\partial \chi}{\partial N}\left(N-N_{0}\right)+\frac{\partial \chi}{\partial T}\left(T-T_{0}\right)
$$

Wo assume that the effects of the constant $x\left(N_{0}, T_{0}\right)$ are absorbed into the avity decay constant $k$. The above expression can subsequently be cast into the following form by introducing the density and temperature $\alpha$-factors [10]:

$$
x(N . T)=-i \alpha_{v}\left(N-N_{0}\right)\left(1-i \alpha_{N}\right)-i \alpha_{T}\left(T-T_{0}\right)\left(1-i \alpha_{T}\right)
$$


A few words about the model are in order. To start from a more fundamental level, we need to solve the Boltzmann equations coupled to the optical field and TDF as well as to the optical polarization. This would correspond to the semiconductor Bloch equations (SBEs)[11] with an additional $\mathrm{TH}_{z}$ field. The coupling of such SBEs with the wave equation for the optical field will offer a more complete framework for studying the interplay between the TDF, optical field, charge carriers, and phonons in a semiconductor. For lasers, a systematic approximation along the line of moment equations would be more feasible. Such an investigation is currently being pursued and the results will be reported elsewhere.

()ur present approach, represented mainly by Eqs. (2)-(3) can be justified in the following way. First, we ignore the heating effect due to stimulated emission of optical photons, as this heating is much less important for lasers slightly above threshold than heating due to the TDF. This allows a sinplification of the coupling between the heating and lasing processes; heating due to the $\mathrm{TH} z$ field will influence lasing, but not visa versa. Under this approximation, the set of moment equations will be reduced to Eqs. (2) and (3) if we further assume that the relaxation of the average momentum and cucrgy is much faster than the relaxation of the total carrier density and laser field. Due to this simplification, we can solve $\mathrm{Eq}$. (1) for a given carrier density separately. The resulting distribution will be fitted to a Fermi function which thus determines the plasma temperature at a given density under the influence of the TIIz ficld. Such a calculation allows us to obtain the temporal variation of the plasma temperature on the time scale of the TDF. The underlying assumption is that carriers after accelerating will thermalize quickly through c-c and c-ph scattering and a well defuned temperature will oscillate with the TDF. This is a valid assumption due to the subpicosecond 
scattering processes and is also established experimentally in Ref. [6].

Figure 1 shows typical examples of the temperature evolution by application of TDFs with an amplitude of $2.5 \mathrm{kV} / \mathrm{cm}$ (circles) and $5.0 \mathrm{kV} / \mathrm{cm}$ (dashed lines), respectively. We see that after a rapid initial temperature increase from $30 \mathrm{~K}$ (our chosen lattice temperature), the plasma temperature is stabilized within $\sim 1 \mathrm{ps}$ to a sinusoidal form. The time dependence of the temperature as a solution of the Boltzmann equation after the initial transients can be fitted to the form:

$$
T=T_{0}\left(F_{T H_{z}}\right)+T_{a m n}\left(F_{T H_{z}}\right) \cos \left(4 \pi \nu_{0} t+\phi_{0}\right)
$$

where $\nu_{0}$ is the frequency of the TDF. As shown by the solid line in Fig. 1. the fitting is almost indistinguishable from the actual data. Similar behavior for a stronger field $\left(F_{0}=5.0 \mathrm{kV} / \mathrm{cm}\right)$ is depicted by the dashed curve. This time dependence of the plasma temperature is consistent with those seen in another theoretical work [8]. The $T_{0}$ and $T_{a m}$ values are similar to those measured experimentally and calculated theoretically.

In the following; we assume that the time dependence of the plasma temperature is given by Eq. (6). To study the effects of the time-dependent plasma temperature, we solve Eqs. (2)-(3) numerically for a typical edge emitting diode laser. As a first example, we consider a laser of $200 \mu \mathrm{m}$ in length with cleaved facets at both ends. The power reflectivity is assumed to be $35 \%$ fur both facets. The active region consists of $3 \mathrm{QWs}$ of $10 \mathrm{~nm}$ in width. The values of the $\alpha$-parameters are taken from Ref. [10]. In all our examples, we use the values of $T_{0}=130 \mathrm{~K}$ and $T_{a n}=40 \mathrm{~K}$. Figure 2 shows the laser intensity (solid lines) and carrier density (dashed lines) for two (asse's. In Fig. 2(a), $F_{\mathrm{THz}}$ is modulated at $50 \mathrm{GH}$, while the corresponding frequency of the laser intensity is $100 \mathrm{GHz}$. Wo recognize from the figure 
that there is a regular sinusoidal oscillation with a deep modulation, while the carrier density shows only a slight wiggle. In Fig. 2(b), the frequency is increased to $0.5 \mathrm{THz}$. The intensity yields the same regular oscillation, but the modulation depth is significantly smaller than in Fig. 2(a); additionally, the carrier density is now a constant over many oscillation cycles, indicating that the slow interband recombination is not involved in this fast modulation.

With a further increase in the frequency of the TDF, the same regular intensity oscillations are observed. The modulation depth, however, decreases. For a frequency of a few $\mathrm{THz}$, our model needs to be modified and will be discussed in a future investigation. In Fig. 3 two examples of low frequency TDFs are shown. As the temperature oscillation frequency decreases, the coupling with the carrier dymamies becomes important since there is a near resonant interaction between the plasma temperature oscillation and the relaxation oscillation which is of the order of a few GHz. Figure 3(a) clearly shows signatures of such an effect. Consequently, the intensity-oscillation pattern is modified and the carrier density changes appreciably during the intensity oscillation. For the slightly higher temperature-oscillation frequency in Fig. 3(b) (25 GIIz), we see a deeper modulation. Now the laser is almost switched off. The intensity pattern of Fig. 3(b) also shows a period-cloubling phenomenon. This is again caused by an interplay between the carrier and temporature dynamics.

To conclude, we have illustrated an ultrafast scheme to modulate and switch semiconductor lasers at $\mathrm{TH} z$ and sub-THz frequency rates. This is possible by circumventing the usual slow interband carrier dynamics. We show that under a sinusoidal TDF, the temporal pattern of the electrical field can be converted to a similar, (quite regular pattern of the laser intensity. This faithful representation of the sinusoidal THz field by a laser intensity pattern 
in the visible or infrared domain may offer an alternative way of measuring high-frequency alternating electrical fields. For lower-frequency oscillation, the same arrangement can lead to an almost $100-\%$ modulation and even on-off switching behavior. This could be useful for switching semiconductor lasers. Currently we are conducting a more detailed study using the moment equations mentioned above.

\section{Acknowledgment}

The work at NASA Ames Research Center is supported by the Director Discretionary Fund (DDF) and by the NASA contract NAS2-14303. The work at IVSU was supported by the National Science Foundation by grant DMR9705403 and by the Office of Naval Research. The authors thank Jianzhong Li for useful discussions.

\section{References}

[1] G.P. Agrawal and N.K. Dutta, Semiconductor Lasers, Van Nostrand Reinhold, New York, 1993

[2] A. Yariv, Optical Electronics in Modern Communications, (Oxford University Press, 1997)

[3] F. Jahnke and S.W. Koch, Appl. Phys. Lett., 67, 2278(1995)

[4] S.G.Hense and M. Wegener, Phys. Rev. B 55, 9255 (1997)

[5] M. Elsaesser, S.G. Hense, and M. Wegener, Appl. Phys. Lett., 70, 853(1997) 
[6] N.G. Asmar et al., Appl. Phys. Lett., 68, 829(1996)

[7] W.Xu and Z. Zhang, Phys. Rev. B, 55, 5259(1997)

[8] X.L.Lei, B. Dong; and Y. Q. Chen, Phys. Rev. B 56, 12120 (1997)

[9] S. Hughes and D.S. Citrin, Phys. Rev. B, 58, R15969 (1998)

[10] C.Z. Ning: Appl. Phys. Lett., 72, 1887(1998)

[11] W.W.Chow, S.W.Koch, and M.Sargent, Semiconductor-Laser Physics. Springer, New York, 1995

\section{Figure captions}

Fig. 1 Plasina temperature oscillation by means of a terahertz driving field at two field amplitude values: $2.5 \mathrm{kV} / \mathrm{cm}$ (circles) and $5.0 \mathrm{kV} / \mathrm{cm}$ (dashed lines).

Fig. 2 Laser intensity and carrier density versus time in the presence of a terahert/ driving field of frequency $50 \mathrm{GHz}$ (a) and $500 \mathrm{GHz}$ (b)).

Fig. 3 As in Fig. 2 but with a ficld frequency of $5 \mathrm{GHz}$ (a) and $25 \mathrm{GHz}$ (b). 

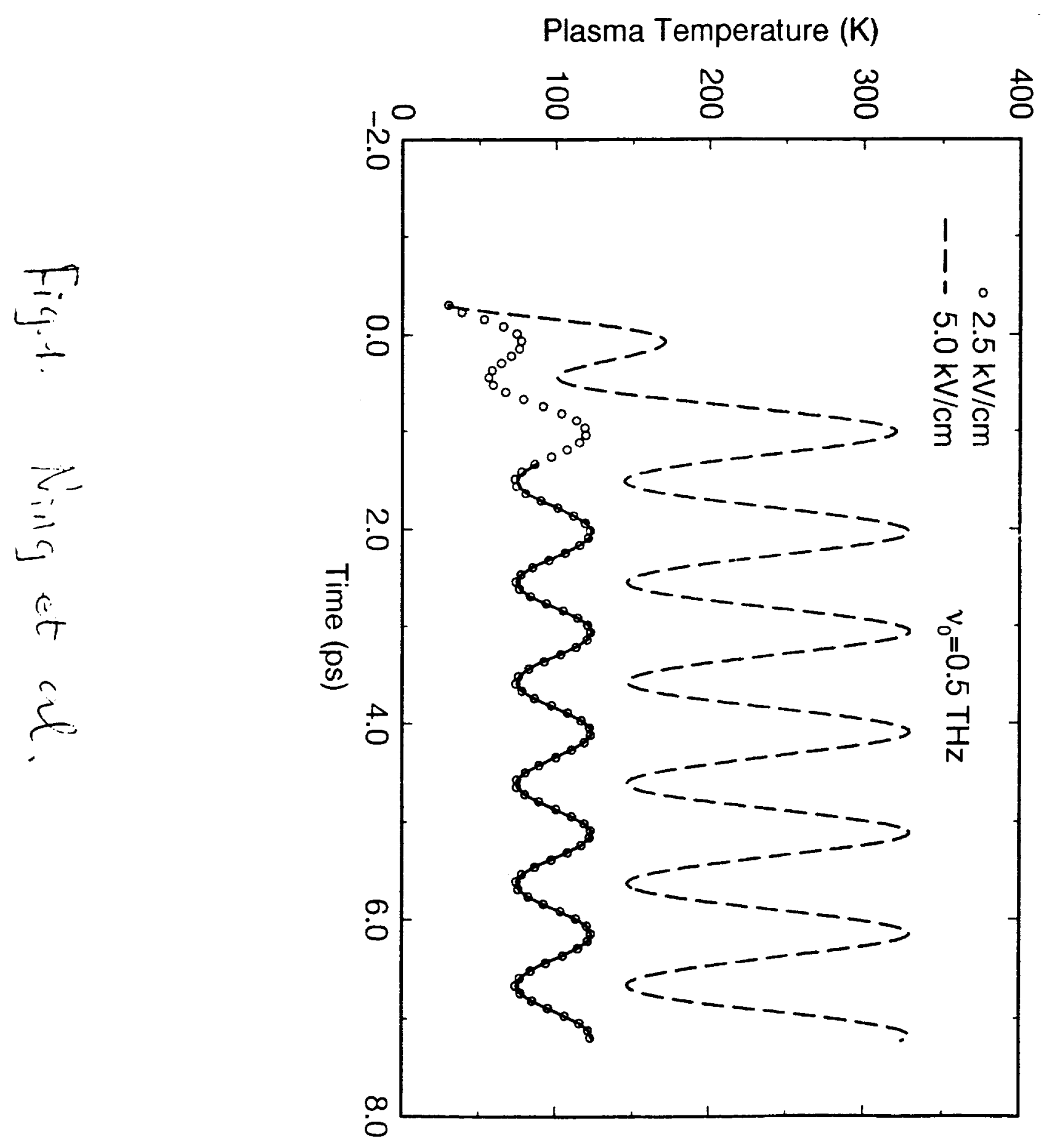


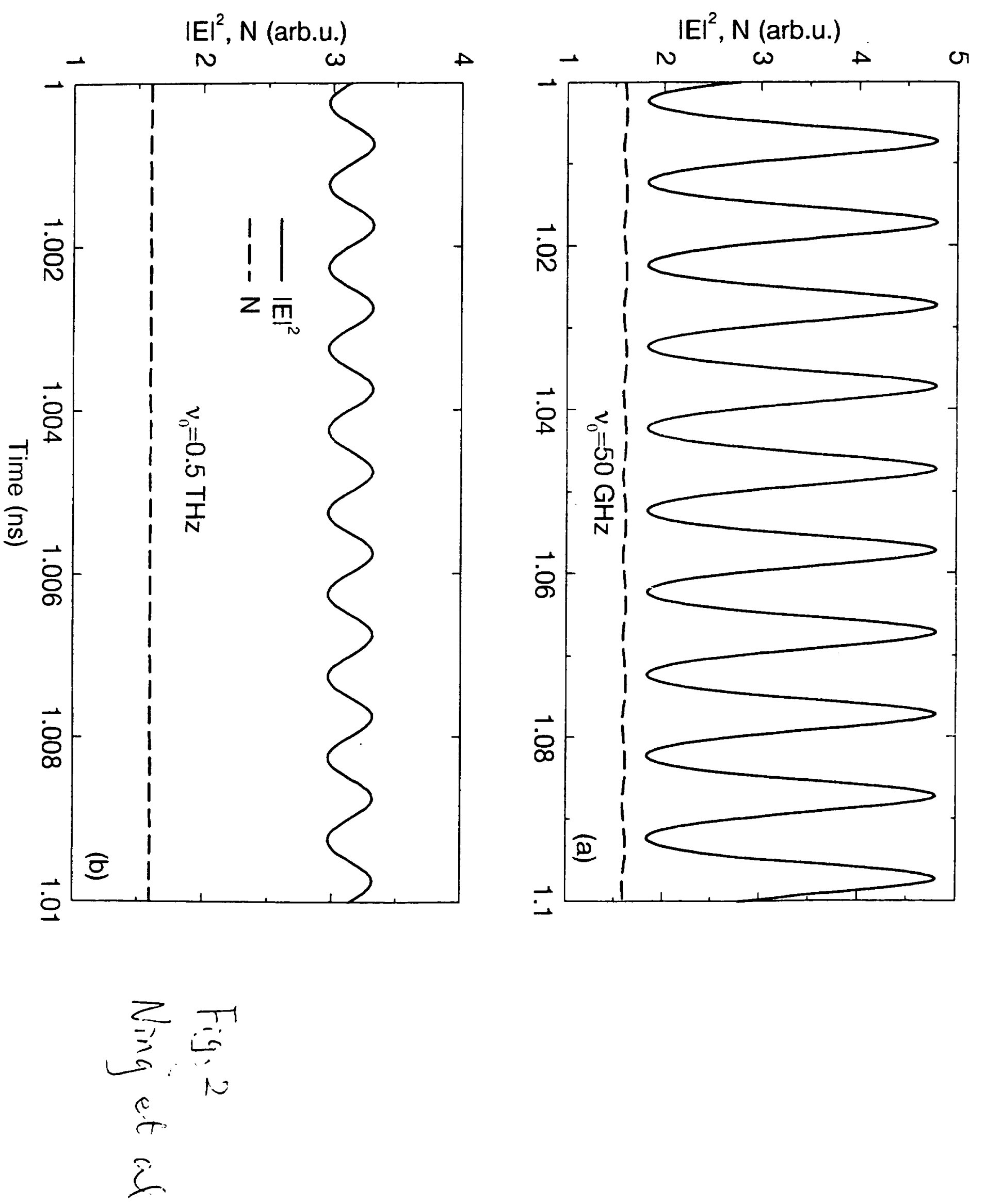



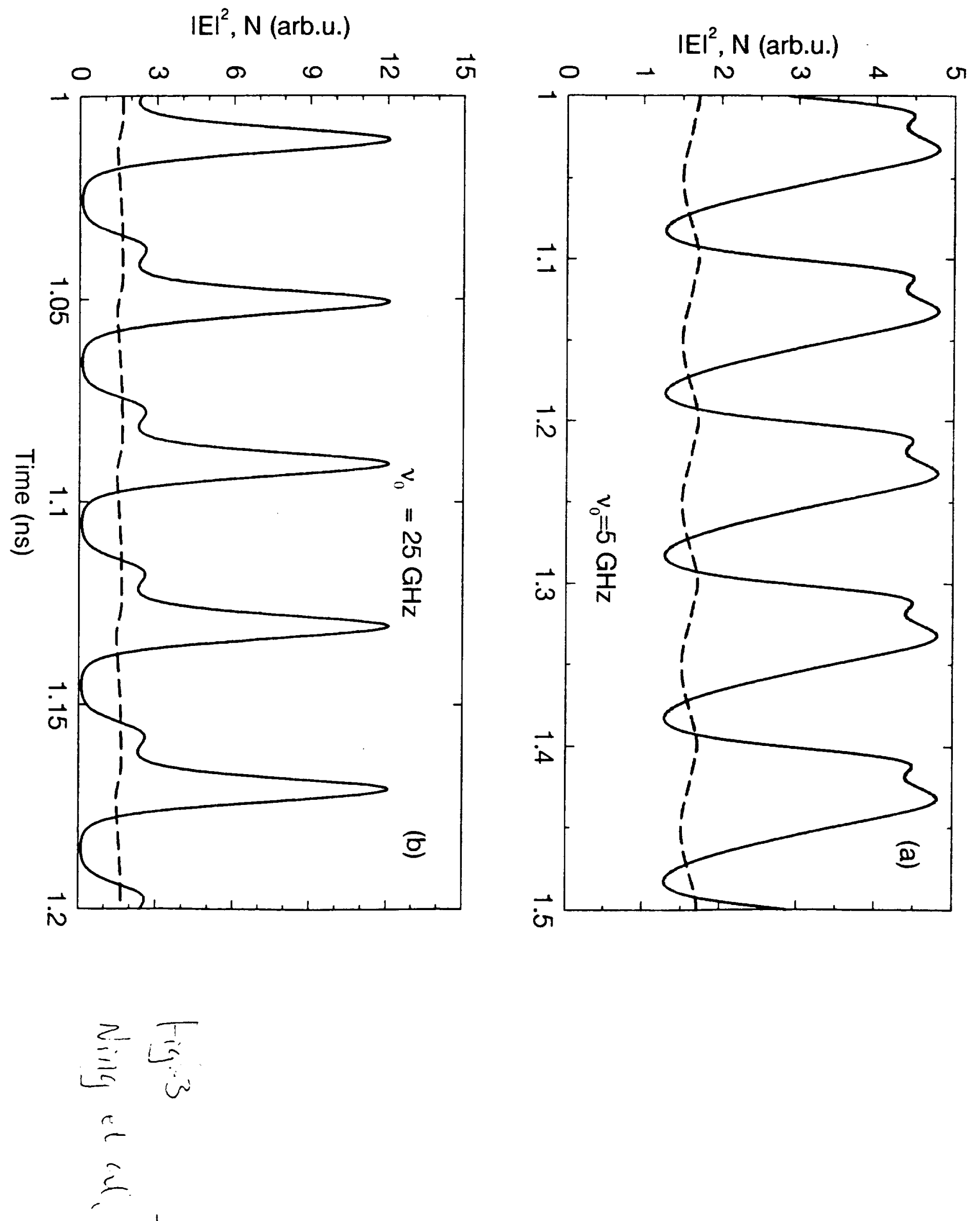